\title{
DECOUPLED STEADY-STATE MODEL OF THE MODULAR MULTILEVEL CONVERTER WITH HALF-BRIDGE CELLS
}

\author{
Staffan Norrga *, Lennart Ängquist*, Kalle Ilves*, Lennart Harnefors ${ }^{\dagger}$ and Hans-Peter Nee* \\ *KTH Royal Inst of Technology, Sweden,norrga@kth.se, ${ }^{\star} A B B$ AB, Sweden.
}

Keywords: Cascaded Converter, Modular Multilevel Converter, Frequency domain

\begin{abstract}
Modular multilevel converters, based on cascading of halfbridge converter cells, can combine low switching frequency with low harmonic interference. They can be designed for high operating voltages without direct series connection of semiconductor elements. This has led to a rapid adoption within high-power applications such as HVDC, STATCOM and railway interties. Analysing the operation of these converters in the frequency domain poses a few challenges due to the presence of significant low-order harmonic voltages in the cell capacitors. This paper presents a frequency-domain model of the MMC converter with halfbridge cells, based on a two-stage approach. First, the circuit equations are decoupled by a simple linear transformation, whereby the circuit schematic can be separated into a dc-side and an ac-side part. Second, the switching operation within the phase arms is modelled in the frequency domain by iterated convolution. The model is verified against a timedomain simulation of a converter with ratings valid for HVDC applications. It is shown that the proposed methodology, where all calculations are made in the frequency domain, can accurately reproduce the results from the simulation.
\end{abstract}

\section{Abbreviations and Nomenclature}

\subsection{Abbreviations}

HVDC High voltage direct current

NPC Neutral point clamped converter

MMC Modular multilevel converter

\subsection{Symbols}

U Voltage

I Current

C Cell capacitance

$L_{b} \quad$ Phase branch inductance

$Z_{b} \quad$ Phase branch impedance

$M \quad$ Modulation index

For time-varying quantities, upper-case symbols denote frequency domain representations whereas lower-case symbols denote time domain signals.

\subsection{Indices and subscripts:}

$\begin{array}{ll}n & \text { Cell number within a string } \\ h & \text { Harmonic order } \\ k & \text { Summation indices } \\ u & \text { Upper phase branch } \\ l & \text { Lower phase branch } \\ p & \text { Difference of phase-branch quantities } \\ c & \text { Average of phase-branch quantities } \\ d & \text { Dc-side quantities } \\ a & \text { Ac-side quantities } \\ i & \text { Phase leg number } \\ \text { cap } & \text { Cell capacitor }\end{array}$

\section{Introduction}

The modular multilevel converter (MMC) based on halfbridge cells [1], see Figure 1 is rapidly attracting increased interest and is being considered for a number of applications ranging from HVDC transmission [3][4] to medium-voltage motor drives [5]. The topology offers the significant benefit compared to previous multilevel converter circuits, such as two-level and NPC (neutral point clamped) topologies, that the number of levels in the output voltage can be varied without making the circuit excessively complex. The number of levels can be increased simply by adding more cells to the phase branches, so that tens or even hundreds of levels are conceivable. The basic structure of the simple two-level converter with three phase legs is remains, but the static valves are replaced by phase branches consisting of cascaded cells and inductors. This also allows for the possibility to design high-voltage converters handling hundreds of kilovolts without direct series connection of the power semiconductor elements [2]. The fact that a large number of voltage levels can be achieved implies that the harmonic requirements on dc side as well as ac side can be fulfilled with a minimum of filtering even at very low switching frequency. In some applications the switching frequency can be reduced to only a few times the fundamental frequency, whereby it has been possible to reduce the losses significantly compared to earlier designs based on two- and three-level converters. However, the nature of these converters implies a few drawbacks. One is that the current fed into the cell capacitors will contain lowfrequency modulation products (mainly first and second harmonic). This implies that the capacitor voltage ripple will contain the corresponding harmonics, whereby the required total capacitive energy storage needs to be considerably larger 


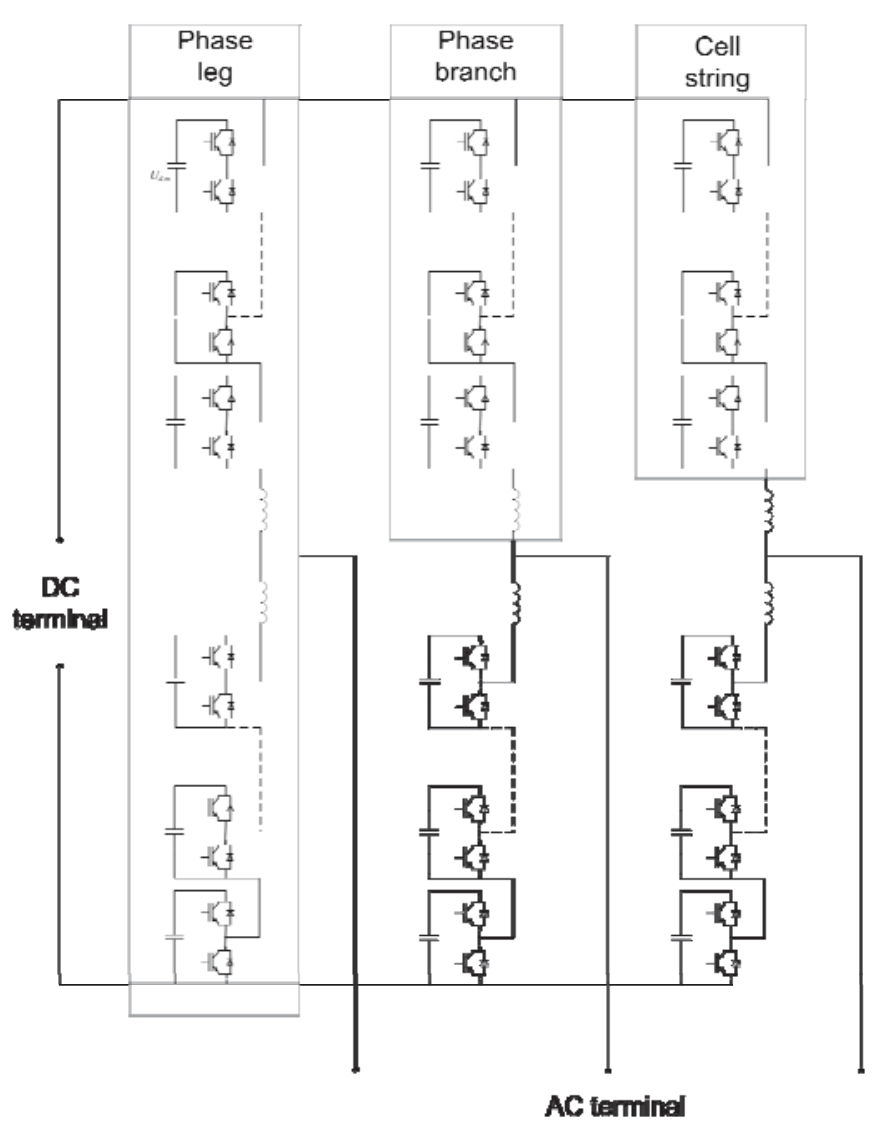

Figure 1: Circuit schematic of a modular multilevel converter with half-bridge cells indicating the various functional parts.

than in a conventional two-level or three-level NPC converter. To some extent, the harmonic distortion difficulties experienced with the previously used two-and three-level topologies are replaced by harmonic fluctuation challenges in the cell capacitors which require attention with regard to the dimensioning and control of the system. An analytical study is made in Ref. [6] of the impact of capacitor voltage ripple, under simplified assumptions with regard to the control and modulation of the converter.

To date however, there has been no comprehensive frequency-domain model of this topology and the analysis has generally relied on extensive simulation studies. In this paper a steady-state harmonic model is presented which can explain the relationship between the operating point variables, the pulse pattern used in the cells, and the harmonics of the cell capacitor voltages and the circulating currents.

\section{Decoupled circuit model}

In this section the circuit equations governing the converter are decoupled by using a simple linear variable transformation linking the currents and voltages of the phase branches to quantities that are more convenient for describing the converter. At this stage the cell strings in each phasebranch are treated as voltage sources.

In Figure 2 an equivalent circuit of the converter is displayed. The cell chains of the phase branches are, as mentioned, modelled as voltage sources with voltage $u_{l i}$ (lower branch, phase leg $i$ ) and $u_{u i}$ (upper branch, phase leg $i$ ). The corresponding currents flowing through the phase branches are labelled $i_{l i}$ and $i_{u i}$. The external circuits on the ac and dc sides are modelled by simple Thevenin equivalents allowing for common mode as well as differential mode voltages and currents on both sides. The mentioned linear transformation used is as follows:

$$
\begin{gathered}
U_{p i}=\frac{U_{l i}-U_{u i}}{2} \\
U_{c i}=\frac{U_{u i}+U_{l i}}{2} \\
I_{p i}=I_{u i}-I_{l i} \\
I_{c i}=\frac{I_{u i}+I_{l i}}{2} .
\end{gathered}
$$

A physical interpretation of these quantities is that $I_{p i}$ are the ac-side phase currents and $U_{p i}$ the inner emf:s of each phase leg driving these currents. Correspondingly, $I_{c i}$ are the average currents of each phase leg, whereas $U_{c i}$ are the voltages driving these currents. The main part of these latter voltages and currents is the DC component, accounting for the active power flow through the converter. However $I_{c i}$ also contains any currents circulating between the phase legs and $U_{c i}$ any voltages driving these circulating currents.

The inverse of the transformation is obtained simply by solving for the phase-branch quantities:

$$
\begin{gathered}
I_{u i}=I_{c i}+\frac{I_{p i}}{2} \\
I_{l i}=I_{c i}-\frac{I_{p i}}{2} \\
U_{l i}=U_{c i}+U_{p i} \\
U_{u i}=U_{c i}-U_{p i}
\end{gathered}
$$

The voltage equation for a loop comprising an arbitrary phase leg and the dc link can be written as follows:

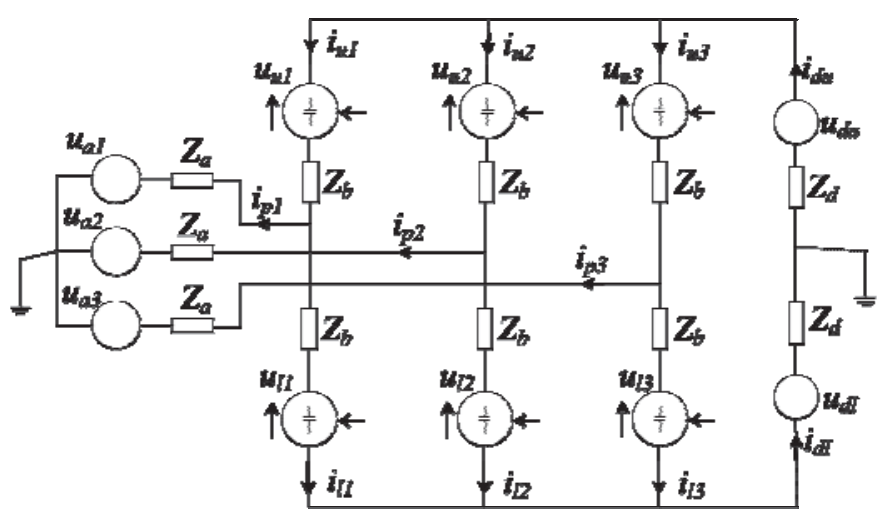

Figure 2: Equivalent schematic of the converter modelling the cell strings as voltage sources. 


$$
U_{u i}+U_{l i}+Z_{b} I_{u i}+Z_{b} I_{l i}-U_{d u}-U_{d l}+Z_{d} I_{d u}+Z_{d} I_{d l}=0
$$

Substituing the definitions (5) through (8) into this equation and simplifying yields

$$
U_{d c}-Z_{d} I_{d c}-U_{c i}-Z_{c} I_{c i}=0
$$

Correspondingly, the voltage equations for a loop going from the midpoint of the DC link to the midpoint of the ac side reads as follows:

$$
U_{d u}-Z_{d} I_{d u}-U_{u i}-Z_{b} I_{u i}-Z_{a} I_{p i}-U_{a i}=0
$$

and

$$
-U_{d l}+Z_{d} I_{d l}+U_{l i}+Z_{b} I_{l i}-Z_{a} I_{p i}-U_{a i}=0
$$

By summing these two equations and substituting the employed linear transformation (Eq. (5) through (8)) into the resulting equation it is found that:

$$
U_{p i}-\frac{Z_{b}}{2} I_{p i}-Z_{a} I_{p i}-U_{a i}-U_{d p}-\frac{Z_{d}}{2} I_{d p}=0 .
$$

Finally, applying Kirhhoffs current law to the positive and negative dc rails, respectively, of the circuit in Figure 2 we obtain

$$
\begin{gathered}
I_{d u}=I_{u 1}+I_{u 2}+I_{u 3} \\
I_{d l}=I_{l 1}+I_{l 2}+I_{l 3} .
\end{gathered}
$$

Summing and subtracting these equations, respectively, and employing the definitions given by (5) and (6) we get:

$$
\begin{gathered}
I_{d c}=I_{c 1}+I_{c 2}+I_{c 3} \\
I_{d p}=I_{p 1}+I_{p 2}+I_{p 3} .
\end{gathered}
$$

Eq. (11) and Eq. (15), on the one hand, correspond to an equivalent schematic governing all ac-side currents and the common-mode dc-side current, shown in Figure 3(a).

Eq. (10) and Eq. (14), on the other hand, correspond to an equivalent schematic governing the circulating currents and the differential-mode dc-side current; see Figure 3(b). The dominant part of the latter current is generally the $\mathrm{dc}$ component, which accounts for the active power flow between the dc and ac sides.

\section{Power conversion within the cell strings}

In the previous section it was assumed that the cell strings of the phase legs can be treated as voltage sources. Due to the presence of low-frequency voltage and current harmonics in the cell capacitors this assumption is only applicable with a few qualifications. To gain a better understanding of the behaviour of the cells and cell strings when the cell capacitor ripple cannot be neglected a frequency-domain model is derived in this section. Throughout the section as well as the next section, only one phase leg is considered since the treatment of all phase legs is identical. For this reason the index $i$, related to the phase leg number is dropped. Figure 4 shows a cell, numbered $n$, with the relevant electrical quantities indicated. The signal $s_{\left\{u, l_{i} n\right.}$ is the switching function which assumes the value 0 when the valve in parallel to the cell terminal conducts and the value 1 when the valve in series with the capacitor conducts.

Firstly, the equations relating the cell capacitor voltage and current to the voltage and current at the cell terminal are written:

$$
\begin{gathered}
u_{\{u, l\}, n}=s_{\{u, l\}, n} u_{\{u, l\}, c a p, n} \\
i_{c a p,\{u, l\}, n}=s_{\{u, l\}, n} i_{\{u, l\}, n} .
\end{gathered}
$$

Eqs. (16) and (17) imply time-domain multiplications, which cannot be described by linear circuit theory. A time-domain multiplication of two periodic signals $\mathrm{x}$ and $\mathrm{y}$,

$$
f(t)=x(t) y(t),
$$

corresponds in the frequency domain to a convolution of the Fourier coefficients of the signals:

$$
F_{h}=\sum_{k=-\infty}^{\infty} X_{k} Y_{h-k}
$$

Capitalised symbols denote the complex Fourier coefficients of the corresponding time-domain signals i.e.

(a) Ac-side circuit (Describes ac side PS/NS/ZS and DC side common mode)

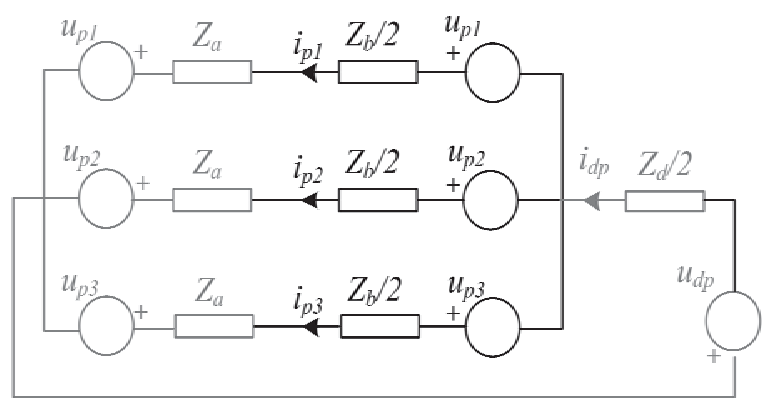

(b) Dc-side circuit

(Describes circulating currents and dc side differential mode)

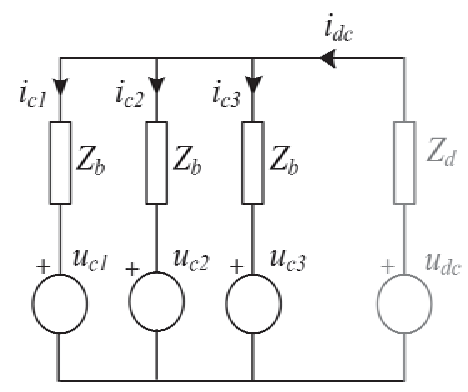

Figure 3: Decoupled equivalent circuits of the converter for the ac and dc sides. Parts related to the converter highlighted. 


$$
X_{h}=\int_{t}^{t+T} x(t) e^{-j 2 \pi h t / T} d t
$$

with $T$ being the cycle time and $h$ being the harmonic order. For numerical calculations, the number of terms in the Fourier series expansions is obviously always limited.

The nonlinearity of the switching function in the cells provides a few challenges for the derivation of a frequency domain model. According to Eq. (17) the capacitor current, and hence the capacitor voltage ripple, will contain modulation products of the switching function and the phasebranch currents. On the other hand, Eq. (16) shows that the capacitor voltage ripple will influence the voltage at the cell terminals, and hence also the phase-branch currents, according to the discussion in the previous section. Thus, the branch currents influence the capacitor voltages which in turn affect the cell output voltages that together contribute to the phase-branch currents etc. For this reason, it is not trivial to obtain a closed analytical solution to the harmonic problem in the general case. A possible approach is to iterate between calculation of the capacitor harmonics on the one hand, and the phase-branch currents on the other hand until a solution is found. Such an algorithm is outlined in Figure 5, and described in detail below.

Starting from an estimate of the phase-branch current, the harmonic components of the current injected into cell capacitor $n$ can be obtained as

$$
I_{c a p}\{u, l\}, n=S_{\{u, l\}, n} * I_{\{u, l\}}
$$

where the symbol * denotes discrete convolution according to Eq. (19). Secondly, each harmonic of the capacitor voltage can be computed as

$$
U_{c a p\{u, l\}, n, h}=\frac{I_{c a p\{u, l\}, n, h}}{j \omega_{0} h C}
$$

It should be noted that this expression is not applicable to the dc component. The dc component of the capacitor voltage is related to the energy balance of the converter which the steady-state model does not take into account directly. However, it has to be ensured that the dc component of the capacitor current is zero, since stable operation is impossible otherwise. The dc component is set to a value consistent with the control of the converter, see sect 5 for a further discussion on the choice of this value.

In the next stage the output voltage of the cell is computed by again convolving with the switching function as follows:

$$
U_{\{u, l\}, n}=S_{\{u, l\}, n} * U_{\operatorname{cap}\{u, l\}, n} .
$$

The overall cell string voltage can now be obtained by summing over the cell voltages:

$$
U_{\{u, l\}}=\sum_{n=1}^{N} U_{\{u, l\}, n} .
$$

Having obtained the cell string voltages, the transformation according to Eqs. (1) through (4) is employed, which allows

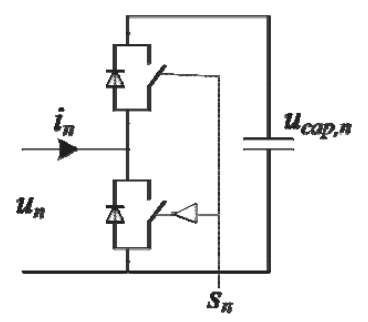

Figure 4: Half bridge cell with switching function.

for use of the decoupled circuit model in Figure 3 to compute the current harmonics. In case the converter is connected to a stiff DC link it is obvious that the circuit in Figure 3 (b) is less suitable for determining the dc component of $I_{c}$, which instead has to be set to a value ensuring the energy balance of the converter.

For a further discussion of the energy balance and setting the dc-component of the dc-side current, see next section.

Finally, applying the inverse transformation (5) - (8) yields the phase-branch currents and the procedure can be repeated.

\section{Energy balance}

For the steady-state operation of the MMC converter it is vital to maintain energy balance between the $\mathrm{dc}$ and ac sides. In the previous chapter it was mentioned that the frequency-domain model does not give any direct guidance as to the dc components of the cell capacitor voltages and the converter dc-side current, both of which are related to the energy balance of the system. In this section the considerations necessary for choosing these variables in each iteration are discussed.

As regards the average cell capacitor voltage, a few alternatives are possible, depending on the control of the considered converter. If, on the one hand, a control algorithm is implemented to maintain the average voltage at a certain setpoint, this value is used since this would be the steady-state value. If, on the other hand, the converter is connected to a dc busbar with constant pole-ground voltage $U_{d c}$, the cell capacitor voltage will adapt to the busbar voltage and this corresponding capacitor-voltage dc component should be

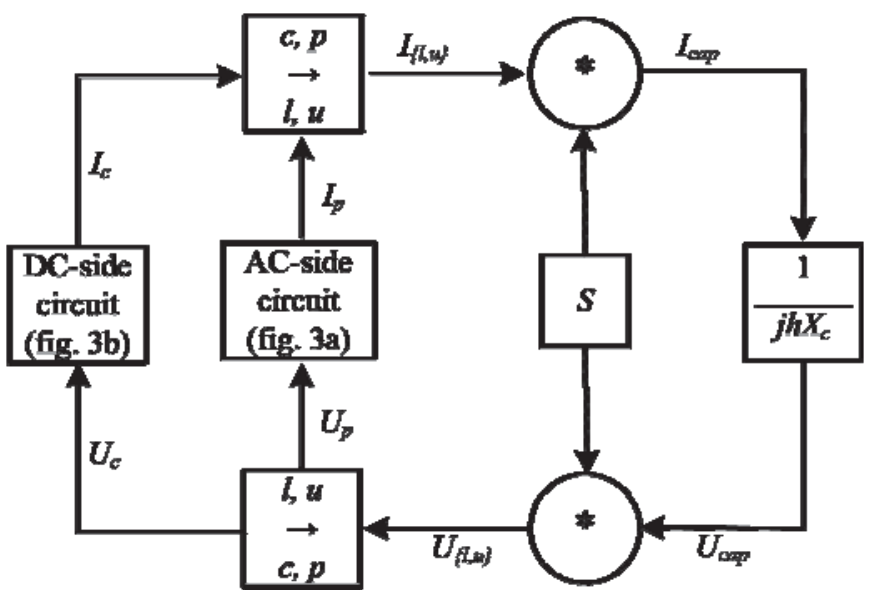

Figure 5: Iterated frequency-domain model of the MMC converter. Indices relating to cell numbers are omitted for clarity. The symbol* denotes convolution. 
determined. In order to do this, the expression for the dc component of the cell string voltages is stated. This is simply the dc component of (23) which can be written

$$
U_{\{u, l\}, n, 0}=S_{\{u, l\}, n, 0} U_{c a p\{u, l\}, n, 0}+2 \sum_{h=1}^{\infty} \operatorname{Re}\left[S_{\{u, l\}, n, h} U_{c a p\{u, l\}, n, h}^{*}\right] .
$$

It is now assumed that the dc components of the capacitor voltages and the switching functions are the same in all cells, whereby the indices $u, l$ and $n$ are dropped from these variables. The natural value for $S_{0}$ is 0.5 , using half bridges, but deviations from this value are also conceivable by introducing a dc component in the reference. Also, the last term (the summation) of Eq. (25) is relabelled $\delta U_{\{u, l\}, n, 0}$. This term corresponds to the deviation of the cell output dc voltage caused by harmonics in the capacitor voltages, and will be significant at reactive power flow. Using Eq. (2) the pole-toground dc voltage imposed by the phase leg can then be written as

$$
U_{c, 0}=N S_{0} U_{c a p, 0}+\frac{1}{2} \sum_{n=1}^{N}\left(\delta U_{l, n, 0}+\delta U_{u, n, 0}\right) .
$$

Observing the schematic in Figure 3(b) and making the fair assumption that the impedances $Z_{b}$ and $Z_{d}$ are largely reactive, we realise that at steady-state $U_{c, 0}=U_{d c}$ has to be fulfilled. Thereby, the required constant component of the cell capacitor voltage can be computed as follows:

$$
U_{c a p, 0}=\frac{1}{N S_{0}}\left\{U_{c, 0}-\frac{1}{2} \sum_{n=1}^{N}\left(\delta U_{l, n, 0}+\delta U_{u, n, 0}\right)\right\} .
$$

The second issue concerning energy balance that could not be resolved by the procedure in the previous section was the determination of the dc-side current $I_{c, 0}$. The ac-side active power flow per phase leg can be computed as

$$
P_{a}=2 \sum_{h=1}^{\infty} \operatorname{Re}\left[U_{p, h} I_{p, h}^{*}\right] \text {. }
$$

Neglecting losses in the converter, the dc-side power should be equal to this value, whereby the average dc-side current is found to be:

$$
I_{c, 0}=\frac{P_{a}}{U_{c, 0}} .
$$

Here $U_{c, 0}$ is determined by Eq. (26). As discussed above, when the converter is connected to a dc busbar it will be forced to be equal to the busbar voltage.

\section{Validation of the proposed methodology}

In this section the proposed methodology is validated by comparison to simulation results from a PSCAD model of a reference converter system, whose main parameters are listed in Table 1. The parameters are representative for a converter to be used in an HVDC application.
Open control is employed, implying that a fixed pwm pattern is used for the switching functions. To synthesize these, carrier-based modulation with phase-shifted carriers is used. Individual triangular carriers are used for each cell and the carrier phase shift is defined by

$$
\theta_{\text {carr }, n}=2 \pi \frac{n}{N}
$$

As long as a noninteger frequency ratio between carrier and reference is used, this method is known not to cause direct imbalances between the cell capacitor voltages. The normalised cell voltage reference is

$$
s_{\{u, l\}, n}^{*}=\frac{1}{2}(1 \pm M) \cos \left(\omega_{0} t+\theta_{0}\right)
$$

for all cells, where the plus-sign applies to the lower branches and the minus sign to the upper branches. The phase shift $\theta_{0}$ is adapted between the phase legs to achieve three-phase symmetry. For this modulation method the harmonic components of the switching function can be found analytically by double Fourier series expansion as described in [7]. In order to maintain periodicity at a non-integer frequency ratio a longer cycle time for the Fourier expansion than the fundamental period of the modulation reference can be used. For the chosen frequency ratio of 3.5 it is appropriate to use a cycle time of twice the reference period. The converter is assumed to be connected to a stiff ac grid via a delta-whye connected transformer, whereby there is no path for zero sequence harmonics between the converter and the grid, i.e. the impedance marked $Z_{b} / 2$ in Figure 3(a) is infinite for these harmonics.

The steady-state model was employed according to the iterative procedure described in Sect 3. and 4. The cell capacitor average voltages and the dc side average current were adjusted in each iteration using Eq. (27) and Eq. (29) from Sect 5, to maintain energy balance. In order to be able to compare the results to those of the time-domain simulation the Fourier series expansions have been evaluated for a few fundamental cycles. Figure 6 and Figure 7 show this type of comparison for the total cell string voltages, and the currents of one phase branch, respectively. The modelled operating point implies nominal ac and dc voltages and purely active power flow from the converter to the ac grid amounting to $880 \mathrm{MW}$. As evident from the graphs, the proposed model can accurately reproduce the simulated waveforms. The reason for choosing the branch quantities for the comparison is that these contain harmonics present in both the circulating

\begin{tabular}{|l|c|c|}
\hline Quantity & Value & $p u$ \\
\hline Number of cells per branch & 36 & - \\
Grid frequency & $50 \mathrm{~Hz}$ & 1 \\
Ac grid voltage, 1-1 & $360 \mathrm{kVrms}$ & 1 \\
Ac current & $1300 \mathrm{Arms}$ & 1 \\
Dc voltage & $\pm 320 \mathrm{kV}$ & - \\
Phase branch reactors & $76 \mathrm{mH}$ & $15 \%$ \\
Cell capacitance & $1 \mathrm{mF}$ & $(28 \mathrm{~kJ} / \mathrm{MVA})$ \\
Transformer leakage reactance & $76 \mathrm{mH}$ & $15 \%$ \\
Switching frequency & $175 \mathrm{~Hz}$ & 3.5 \\
\hline
\end{tabular}

Table 1: Main data of the studied converter system. 
currents and ac-side current, and model errors would therefore be easily identified. Figure 8 finally, shows the average capacitor voltage obtained by the two methods. Also in this case good agreement is obtained between the proposed model and the time-domain simulation.

\section{Conclusion}

A frequency-domain model for computing steady-state electrical variables of modular multilevel converters with half bridge cells has been presented. It is evident that the model can accurately reproduce the results obtained by a detailed time-domain simulation. A benefit of the method is that it allows for a clear separation of steady-state and dynamic effects which is not always possible by time-domain simulation. A weakness of the studied method is that no dynamic phenomena can be modelled, so that it is less useful as a tool for designing and evaluating closed-loop control systems, for instance.

In this paper, the methodology was employed to solve the problem where the converter is connected to fixed ac and dc circuits and a fixed pulse pattern applied for the cells. However, also other problems may be solved by the studied model, which will be the topic of coming work.

Furthermore, the methodology has been applied to the MMC converter with half bridges. However it could with modifications also be applied to other similar cell-based topologies, such as converters employing full bridges, where similar cell capacitor ripple effects are present.

\section{References}

[1] Lesnicar A., and Marquardt R., "An innovative modular multilevel converter topology suitable for a wide power range," IEEE PowerTech Conference, 2003.

[2] Dorn J., Huang H., Retzmann D. "A new Multilevel Voltage-Sourced Converter Topology for HVDC Applications," Cigré Session, B4-304, 2008, Paris, France.

[3] Allebrod S., Hamerski R., and Marquardt R., "New transformerless scalable modular multilevel converters for HVDC-Transmission," Power electronics specialists Conference (PESC), 2008.

[4] B Jacobson, P Karlsson, G Asplund, L Harnefors, T Jonsson, "VSC-HVDC Transmission with Cascaded Two-Level Converters", CIGRE General Meeting 2009.

[5] M. Hiller, D. Krug, R. Sommer, and S. Rohner, "A new highly modular medium voltage converter topology for industrial drive applications," European Power Electronics Conference (EPE), 2009.

[6] Ilves K, Antonopoulos A, Norrga S, Nee H-P, "SteadyState Analysis of Interaction Between Harmonic Components of Arm and Line Quantities of Modular Multilevel Converters", IEEE transactions on power electronics, Vol. 27, No 1, pp. 57-68, Jan 2012.

[7] Bowes S. R., Bird B. M., "Novel Approach to the Analysis and Synthesis of Modulation Processes in Power Convertors", Proceedings of the IEE, vol. 122, No. 5, pp. 507-513, May 1975.
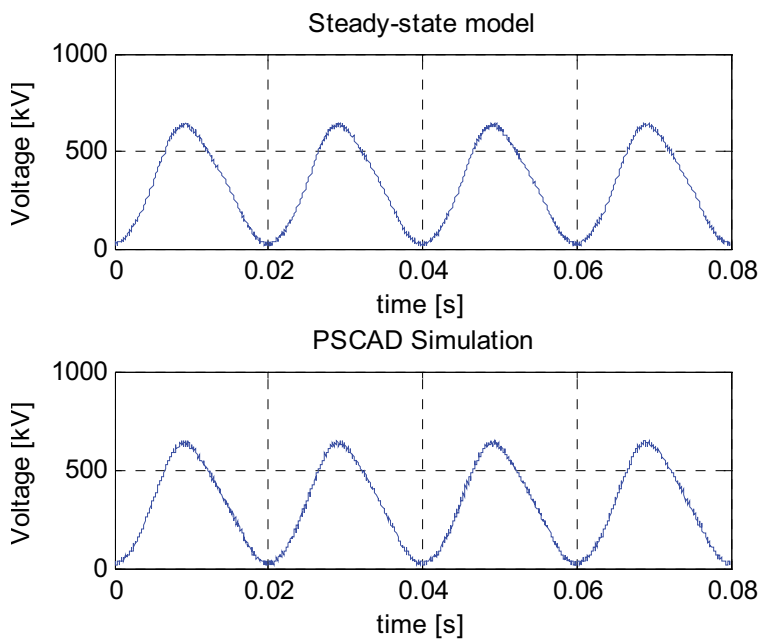

Figure 6: Comparison of cell string voltages for one arm $\left(u_{l}\right)$ obtained with the proposed method (top) and with a PSCAD simulation model (bottom).
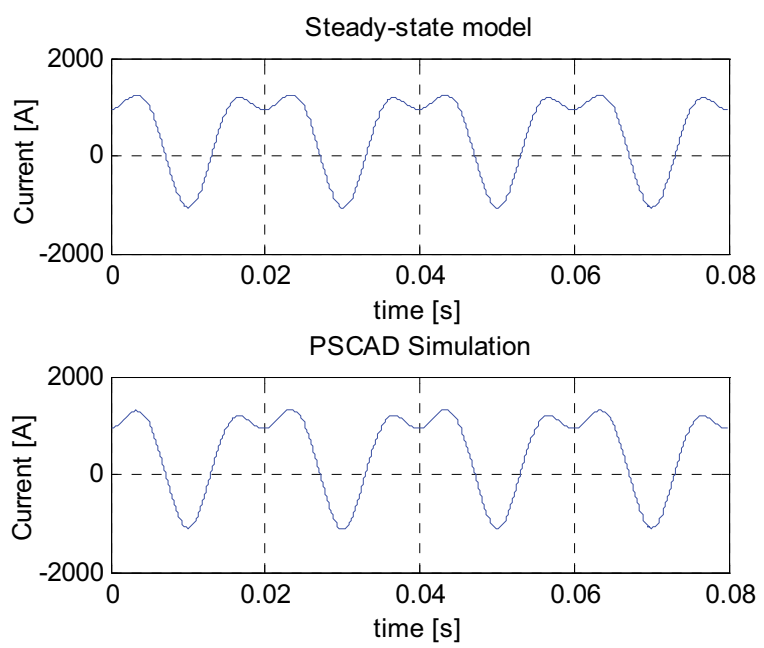

Figure 7: Comparison of phase-branch currents $\left(i_{l}\right)$ obtained with the proposed method (top) and with a PSCAD simulation model (bottom).


Figure 8: Comparison of average capacitor voltage obtained with the proposed method (top) and with a PSCAD simulation model (bottom). 